\title{
On data preprocessing for subspace methods
}

\author{
Dietmar Bauer \\ Department of Electrical Engineering \\ Linköping University, SE-581 83 Linköping, Sweden \\ WWW: http://www.control.isy.liu.se \\ Email: Dietmar.Bauer@tuwien.ac .at
}

June 6,2000

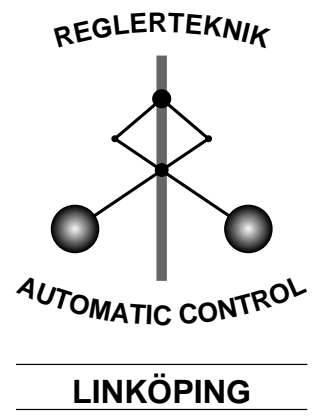

Report no.: LiTH-ISY-R-2265

Submitted to CDC'2000, Sydney, Dec. 2000

Technical reports from the Automatic Control group in Linköping are available by anonymous ftp at the address ftp.control.isy.liu.se. This report is contained in the file 2265.pdf. 



\title{
On data preprocessing for subspace methods
}

\author{
Dietmar Bauer * \\ Institute f. Econometrics, Operations Research and System Theory, \\ TU Wien, Argentinierstr. 8, A-1040 Wien
}

June 6, 2000

\begin{abstract}
In modern data analysis often the first step is to perform some data preprocessing, e.g. detrending or elimination of periodic components of known period length. This is normally done using least squares regression. Only afterwards black box models are estimated using either pseudo-maximum-likelihood methods, prediction error methods or subspace algorithms. In this paper it is shown, that for subspace methods this is essentially the same as including the corresponding input variables, e.g. a constant or a trend or a periodic component, as additional input variables. Here essentially means, that the estimates only differ through the choice of initial values.
\end{abstract}

Keywords: subspace algorithms, linear systems, estimation, identification

\section{Introduction}

It has become standard in modern time series analysis to perform some form of preprocessing on the data prior to identification. However often the effects of this preprocessing are not dealt with in the identification phase. As an example consider the analysis of so called subspace methods: Many algorithms have been proposed by different authors, which are all subsumed under the name 'subspace algorithms', as they show certain similarities. To name just the most popular we cite CCA (Larimore, 1983), MOESP (Verhaegen, 1994) and N4SID (Van Overschee and DeMoor, 1994). The properties of these algorithms have been analysed in a number of papers (for references see e.g. Bauer et al., 1999). However all cited references refer to the case, that no preprocessing prior to identification takes place. Also normally some persistency conditions on the input are imposed, which excludes e.g. the constant as an input variable. Therefore essentially it is assumed, e.g. that the only source for a nonzero mean of the output is due to the filtered input. When this does not hold and no preprocessing is performed, a bias will result. If preprocessing is performed the properties of the data change, which has to be taken into account for the estimation of the uncertainties, i.e. for the estimation of the asymptotic variances of the model obtained by the identification step. This in many cases will be straightforward, however it seems to be more convenient to perform both the preprocessing and the actual identification in one step, in order to unify the treatment and to simplify the calculation of the asymptotic variance.

The aim of this paper is to show, that the preprocessing and the estimation phase in fact can be unified by including the constant or similar terms as input variables and use generalised inverses i.e. regularisation techniques in the respective regressions. In fact, it will be shown, that this leads to essentially the same results in the sense, that there is no difference in the attained accuracy, i.e. that the asymptotic distribution of the estimated system are identical. This shows, that some of the preprocessing might be included into the subspace algorithm without any problem, and thus can also be analysed along the same lines.

\footnotetext{
${ }^{*}$ This work has been done, while the author was holding a post doc position at the Department for Automatic Control, University of Linköping, Sweden. The financial support by the EU TMR project 'SI' is gratefully acknowledged.
} 
The organisation of the paper is as follows: In the next section the model set and assumptions are stated. Section 3 then gives a short description of the main steps of the procedure. Section 4 then states the main results of the paper. In section 5 a numerical example is presented and section 6 concludes.

\section{Model set and assumptions}

In this paper we deal with discrete time, finite dimensional, time invariant, state space systems of the form

$$
\begin{gathered}
x_{t+1}=A x_{t}+B z_{t}+K \varepsilon_{t} \\
y_{t}=C x_{t}+D z_{t}+\varepsilon_{t}
\end{gathered}
$$

Here $y_{t}$ denotes the $s$ dimensional observed output, $u_{t}$ denotes the $m$ dimensional observed input, $\varepsilon_{t}$ the $s$ dimensional white noise. $A \in \mathbb{R}^{n \times n}, B \in \mathbb{R}^{n \times m}, C \in \mathbb{R}^{s \times n}, D \in \mathbb{R}^{s \times m}, K \in \mathbb{R}^{n \times s}$ are parameter matrices. The system usually will be described as $(A, B, C, D, K)$. We will assume throughout, that $\varepsilon_{t}$ is i.i.d. with zero mean and variance matrix $\Omega>0$, having finite fourth moments. Throughout we will assume that the system is stable, i.e. $\left|\lambda_{\max }(A)\right|<1$ holds, where $\lambda_{\max }$ denotes an eigenvalue of maximum modulus. It will also be assumed, that the system is strictly minimum-phase, i.e. that $\left|\lambda_{\max }(A-K C)\right|<1$.

Corresponding to the input we will assume, that the input can be partitioned into two parts: $u_{t} \in \mathbb{R}^{m_{i}}$ and $p_{t} \in \mathbb{R}^{m-m_{i}}$. Here $u_{t}$ accounts for the identification input, whereas $p_{t}$ denotes the additional inputs due to the preprocessing. These additional inputs will be restricted to the following choices:

- a constant term, i.e. $p_{t}=1, \forall t$

- a periodic component, i.e. $p_{t, 1}=\sin (\omega t+\phi)$ for known $\omega \in(-\pi, \pi]$ and $\phi$. In this case also the lagged variable $p_{t, 2}=p_{t-1,1}$ has to be included

- a time trend, i.e. $p_{t}=t, \forall t$

These choices include the typical preprocessing like detrending and eliminating periodic components of known periodicity. Naturally, all these terms could be used in combination. Especially the inclusion of the trend makes the inclusion of a constant necessary in order to account for unknown initial effects. The key feature of these inputs is that they are persistent of order one. This is the reason for including the lagged term for the periodic components. It will be clear from the text, why this is needed. Note that in a similar fashion also more complicated preprocessing can be dealt with, as long as it is done using regression onto deterministic processes which are persistent of order one.

Note, that the model as stated is not identifiable for these inputs, which can be seen from a discussion of the constant: Assume that the input has nonzero mean $\mu_{u}$ and the output has mean $\mu_{y}$. Then we obtain

$$
\mu_{y}=D \mu_{u}+\sum_{j=0}^{\infty} C A^{j} B \mu_{u}
$$

Thus it is clear, that the constribution of the constant is equal to $D_{p}+\sum_{j=0}^{\infty} C A^{j} B_{p}$, where $B_{p}$ and $D_{p}$ denote the columns of $B$ and $D$ respectively corresponding to the constant. This leads to a linear equation in $B_{m}$ and $D_{m}$ showing the nonidentifiability, which will complicate the analysis of the asymptotic distribution.

In the following we will always distinguish between these two kinds of inputs mainly for the reason of stating assumptions for the identification inputs. The reason is the different nature of the inputs: All preprocessing inputs used above have the characteristic of being perfectly predictable from one observation, i.e. they are persistently exciting of degree one. For the identification inputs however higher degrees of persistency will be required. 


\section{Description of the algorithms}

Subspace procedures have been described in a number of papers. Therefore we restrict ourselves to a short description only. For details see the survey in (Bauer, 1998, Chapter 3). Let $Y_{t, f}^{+}=$ $\left[y_{t}^{\prime}, \cdots, y_{t+f-1}^{\prime}\right]^{\prime}$ denote the stacked vector of outputs, where $f$ is a user defined integer. Similarily define $U_{t, f}^{+}$and $E_{t, f}^{+}$using the inputs and the noise respectively in the place of the outputs. Furthermore let $Z_{t, p}^{-}=\left[y_{t-1}^{\prime}, u_{t-1}^{\prime}, \cdots, y_{t-p}^{\prime}, u_{t-p}^{\prime}\right]^{\prime}$, where $p$ is a user defined integer. Then it is easy to show the following equation:

$$
Y_{t, f}^{+}=\mathcal{O}_{f} \mathcal{K}_{p} Z_{t, p}^{-}+\mathcal{O}_{f} \bar{A}^{p} x_{t-p}+\mathcal{U}_{f} U_{t, f}^{+}+\mathcal{E}_{f} E_{t, f}^{+} .
$$

Here $\bar{A}=(A-K C), \bar{B}=(B-K D), \mathcal{O}_{f}=\left[C^{\prime}, A^{\prime} C^{\prime}, \ldots,\left(A^{f-1}\right)^{\prime}\right]^{\prime}$ and

$$
\mathcal{K}_{p}=\left[[K, \bar{B}], \bar{A}[K, \bar{B}], \ldots, \bar{A}^{p-1}[K, \bar{B}]\right.
$$

Further $\mathcal{E}_{f}$ denotes the block Toeplitz matrix, whose $i$-th block row is equal to

$$
\left[C A^{i-2} K, \ldots, C K, I_{s}, 0^{s \times(f-i) s}\right]
$$

where $I_{s}$ is the $s$-dimensional identity and $0^{a \times b}$ is a $a \times b$ dimensional nullmatrix. Finally $\mathcal{U}_{f}$ denotes the block Toeplitz matrix, whose $i$-th column is equal to

$$
\left[C A^{i-2} B, \ldots, C B, D, 0^{s \times(f-i) m}\right]
$$

This equation is the starting point for all subspace algorithms. An outline can be given as follows:

1. Regress $Y_{t, f}^{+}$onto $Z_{t, p}^{-}$and $U_{t, f}^{+}$in order to obtain estimates $\hat{\beta}_{z}$ and $\hat{\beta}_{u}$.

2. $\hat{\beta}_{z}$ will typically be of full rank, whereas the limit $\beta_{z}$ is of rank $n$. Thus a SVD of $\hat{W}_{f}^{+} \hat{\beta}_{z} \hat{W}_{p}^{-}=$ $\hat{U} \hat{\Sigma} \hat{V}^{\prime}=\hat{U}_{n} \hat{\Sigma}_{n} \hat{V}_{n}^{\prime}+\hat{R}$ is performed leading to an estimate $\hat{\mathcal{O}}_{f}=\left(\hat{W}_{f}^{+}\right)^{-1} \hat{U}_{n}, \hat{\mathcal{K}}_{p}=\hat{\Sigma}_{n} \hat{V}_{n}^{\prime}\left(\hat{W}_{p}^{-}\right)^{-1}$. Here $\hat{W}_{f}^{+}$and $\hat{W}_{p}^{-}$are weighting matrices, which have to be chosen by the user. We will assume throughout that they are nonsingular (a.s.) and converge to some limit $W_{f}^{+}$and $W_{p}^{+}$respectively for $T \rightarrow \infty$. $\hat{\Sigma}_{n}$ contains the $n$ dominant singular values and $\hat{U}_{n}, \hat{V}_{n}$ the corresponding left or right respectively singular vectors.

3. Either the estimates $\hat{\mathcal{O}}_{f}$ and $\hat{\beta}_{u}$ or the estimate $\hat{\mathcal{K}}_{p}$ is used to estimate the system.

In the last step, one has two choices: MOESP and one variant of N4SID use the structure in $\mathcal{O}_{f}$ and $\beta_{u}$ in order to estimate the system matrices, whereas CCA and another variant of N4SID use $\hat{\mathcal{K}}_{p}$ to estimate the state as $\hat{x}_{t}=\hat{\mathcal{K}}_{p} Z_{t, p}^{-}$and then use the system equations (1) in order to obtain estimates of the system.

Now there are two possible ways to include the preprocessing in this framework:

- Regress $y_{t}$ and $u_{t}$ onto $p_{t}$ and let the residuals be denoted as $\tilde{y}_{t}$ and $\tilde{u}_{t}$. This preprocessed data then can be used in the subspace method in order to obtain an estimate of the system $(A, B, C, D, K)$.

- Use $y_{t}$ and $z_{t}=\left[u_{t}^{\prime}, p_{t}^{\prime}\right]^{\prime}$ as the data for the subspace method to obtain an estimate of the system $(A, B, C, D, K)$ of the transfer function relating $u_{t}$ to $y_{t}$.

The aim of the next section is to show that these two procedures deliver essentially identical estimates. 


\section{Main results}

Note, that in the present setup the regression in the first step to obtain the estimates $\hat{\beta}_{z}, \hat{\beta}_{u}$ will have to take into account the singularity of the matrix of the regressors due to the multicollinearity introduced by the process $p_{t}$. For $Z_{t, p}^{-}$note, that in the actual calculation only the vectors $Z_{t, p}^{-, \Pi}$ are used for the estimate $\hat{\beta}_{z}$. Here $Z_{t, p}^{-, \Pi}$ denotes the residuals of $Z_{t, p}^{-}$regressed onto $U_{t, f}^{+}$. Using the notation $\left\langle a_{t}, b_{t}\right\rangle=T^{-1} \sum_{t=p+1}^{T-f} a_{t} b_{t}^{\prime}$ this is equal to

$$
Z_{t, p}^{-}-\left\langle Z_{t, p}^{-}, U_{t, f}^{+}\right\rangle\left\langle U_{t, f}^{+}, U_{t, f}^{+}\right\rangle^{\dagger} U_{t, f}^{+}
$$

Here ${ }^{\dagger}$ denotes the Moore Penrose pseudo inverse. Due to the nature of the variables $p_{t}$, the residuals in all coordinates corresponding to $p_{t}$ are zero. Note that this corresponds to using the preprocessed data $\tilde{y}_{t}, \tilde{u}_{t}$ up to the difference of at most $f+p$ terms in the calculation of the regressions for the preprocessing. This difference is asymptotically negligible under the usual assumptions on $f$ and $p$ to be $o\left((\log T)^{a}\right)$ for some $a<\infty$. Therefore the columns of the estimate $\hat{\beta}_{z}$, which correpond to $y_{t}$ or $u_{t}$ respectively, are equivalent to the ones obtained from preprocessed data. The remaining columns correspond to $p_{t}$ and these are essentially zero (i.e. zero up to effects due to initial values in the regressions). Also note that the calculation of the pseudoinverse could be circumvented by using only those coordinates of $U_{t, f}^{+}$, which are linearily independent. These can be found easily, since the dependence structure of $p_{t}$ is known. In fact, if the regression is performed according to the formula given above, all coordinates corresponding to $p_{t+j}, j>0$ simply can be omitted without changing the result.

In the MOESP type of procedures this shows that the estimates $\hat{\mathcal{O}}_{f}$ of the two alternatives will be essentially identical. This shows the equivalence of the estimates $\hat{A}$ and $\hat{C}$. For the estimation of $B$ and $D$ we consider a special algorithm similar to the MOESP type of procedures: Let $\mathcal{U}_{f}\left(\hat{\mathcal{O}}_{f}\right)$ denote the estimate of $\mathcal{U}_{f}$, where elements of $\mathcal{O}_{f}$ are replaced by their respective estimates. Note that $\mathcal{U}_{f}\left(\hat{\mathcal{O}}_{f}\right)$ is linear in $B$ and $D$. Then the estimate of $B$ and $D$ can be obtained from minimising

$$
\left\|\hat{\mathcal{O}}_{f}^{\perp}\left(\left\langle Y_{t, f}^{+}, U_{t, f}^{+}\right\rangle\left\langle U_{t, f}^{+}, U_{t, f}^{+}\right\rangle^{\dagger}-\mathcal{U}_{f}\left(\hat{\mathcal{O}}_{f}\right)\right)\right\|_{F r}
$$

Here $\hat{\mathcal{O}}_{f}^{\perp} \hat{\mathcal{O}}_{f}=0$ and $\hat{\mathcal{O}}_{f}^{\perp}$ is of full row rank. From this it follows from the block matrix inversion, that the columns corresponding to $u_{t}$ are identical to the respective columns obtained from the preprocessed data $\tilde{y}_{t}, \tilde{u}_{t}$. From this it follows that the estimation of the part of $B$ and $D$, which corresponds to $u_{t}$ is estimated essentially identical as if the preprocessed data would have been used. This shows, that in this case the estimates of the coordinates of the system $(A, B, C, D)$ corresponding to $u_{t}$ is essentially the same as obtained from the preprocessed data. Concerning the part due to $p_{t}$ however the same result does not seem to hold, compare the example given in section 5. This is a matter of further research.

For the Larimore type of approach we have seen that the estimate $\hat{\beta}_{z}$ is essentially identical to the estimate obtained from the preprocessed data in the coordinates corresponding to the output or the input due to $u_{t}$, the other components being zero. Therefore the same holds true for $\hat{\mathcal{K}}_{p}$, noting that for the usual weighting matrices $\hat{W}_{p}^{-}, \hat{W}_{p}^{-}$also only the data $Z_{t, p}^{-, \Pi}$ and $Y_{t, f}^{+, \Pi}$ is used. Here $Y_{t, f}^{+, \Pi}$ is defined analogously to $Z_{t, f}^{-, \Pi}$. These vectors are essentially identical to the corresponding vectors formed from the preprocessed data. As a consequence the state estimate $\hat{x}_{t}=\hat{\mathcal{K}}_{p} Z_{t, p}^{-}$is almost identical to the estimate obtained from the preprocessed data except for differences in the space spanned by the components of $p_{t}$. Note that the next step in this class of algorithms is to use the state in a regression, where these deterministic components are included. This again shows, that the estimate of the system matrices corresponding to $u_{t}$ coincide up to initial conditions with the estimate obtained from the preprocessed data. Note, that the nonidentifiability in this setup is circumvented, as the deterministic components in $\hat{x}_{t+1}$ and $\hat{x}_{t}$ converge to some vectors $\bar{\beta}_{x}$ and $\beta_{x}$ respectively. Letting $\beta_{u}$ denote the coefficients of the deterministic components of $z_{t}$ then it follows from the state equation that

$$
\bar{\beta}_{x}=A \beta_{x}+B \beta_{u}
$$


which determine the components due to $p_{t}$ completely. Therefore the algorithm does not have any nonidentifiability problems by construction and the estimates for $B_{p}$, the columns due to the deterministic components, are consistent and asymptotically normal. After the estimation the nonidentifiability of the model structure could be used to obtain a different normalisation as $B_{p}=0$ using the structure of the nonidentifiability. In both cases the asymptotic distribution will be the same, as if the corresponding estimates have been calculated using the preprocessed data, as is straightforward to see. Finally we have obtained the following theorem:

Theorem 1 Let $y_{t}$ be generated by a system of the form (1), where the noise is i.i.d. with finite moments up to fourth order. Let the input $z_{t}$ consist of two different components: $z_{t}=\left[u_{t}^{\prime}, p_{t}^{\prime}\right]^{\prime}$, where $u_{t}$ is a quasistationary sequence, which is persistently exciting of order $f+p$. Further $p_{t}$ is either a constant term, a trend component or a periodic component with known frequency and shift. Then the estimates of $G_{i}(q)$, the transfer function relating $u_{t}$ to $y_{t}$ obtained by the inclusion of $p_{t}$ in the MOESP type of subspace methods and the estimates obtained by using the residuals from a regression of $y_{t}$ and $u_{t}$ onto $p_{t}$ as the data input for the subspace method without $p_{t}$ are asymptotically equivalent, i.e. the asymptotic distribution of the estimation error are identical.

If the input $u_{t}$ is an ARMA process generated by i.i.d. white noise having a uniformely bounded spectrum and $p=p(T)=o\left((\log T)^{a}\right), p(T) \geq-\frac{d \log T}{2 \log \left|\rho_{0}\right|}$, then the same holds for the Larimore type of algorithms. In this case also the estimates of the transfer function relating the noise to the output will be asymptotically equivalent. Furthermore also the estimates of the effects of the deterministic terms are essentially the same.

It should be noted, that the assumptions of the theorem are by no means necessary. In fact, much weaker conditions suffice. For a discussion on the necessary conditions in the MOESP type of algorithms see (Bauer and Jansson, 2000), for assumptions in a martingale framework for the Larimore type of procedures see (Bauer et al., 1999).

The significance of the theorem is that it gives the user two different but equivalent ways to deal with nonzero means, drifts and periodic components included in many time series. Either one can preprocess the data and estimate and test for the inclusion of these terms beforehand and then use the preprocessed data in order to obtain estimates of the dynamical systems, which are the main goal in many applications. The alternative way is to use the deterministic terms as additional inputs in the subspace method. Both procedures have advantages and disadvantages. The big advantage for the preprocessing approach lies in the fact, that the testing procedures, whether the detmerinistic components are contained in the data at hand, are well established and implemented in many programs. This facilitates the analysis for the user. On the other hand in this case the inclusion of the preprocessing stages in the calculation of the asymptotic variance of the subspace estimates is possible, although cumbersome.

The inclusion of the terms in the estimation procedure is computationally simple. The original procedures can be used, if the regressions are done in a robust way using the pseudoinverses in the case of multicollinearities. The direct embedding of the preprocessing makes the calculation of the variance easier, although at the present time no programs to calculate the asymptotic variance of the subspace procedure exists, that could be used in an industrial context. For the MOESP type of procedures the derivation of the asymptotic distribution of the estimates of the columns of $B$ and $D$ corresponding to $p_{t}$ seems to be complicated. However for the CCA type of estimates the derivation of these distributions is straightforward. Tests for more realistic hypotheses can be obtained from the asymptotic distribution, e.g. whether or not the deterministic components in the output can be explained only through the components occuring in the input.

\section{Numerical Exmaple}

In this section we will present some simple examples, which illustrate the theory given in the last section. The first example is a one state SISO system, which is described by the following matrices:

$$
A=0.5, B=1, C=1, D=1, K=1
$$




\begin{tabular}{|c|c|ccc|}
\hline Method & Sample size & $A$ & $B$ & $D$ \\
\hline MOESP & no pre. & 0.9183 & 1.1498 & 0.0599 \\
& pre. & 0.4983 & 0.9996 & 0.0013 \\
& incl. & 0.4985 & 1.0001 & 0.0013 \\
\hline & corr. & 0.9983 & 0.9980 & 0.9978 \\
\hline CCA & no pre. & 0.8886 & 1.2125 & 0.0710 \\
& pre. & 0.4999 & 0.9994 & 0.0011 \\
& incl. & 0.5002 & 0.9998 & 0.0011 \\
\hline & corr. & 0.9970 & 0.9974 & 0.9974 \\
\hline
\end{tabular}

Table 1: This table shows the mean values of the estimates of $A, B$ and $D$ estimated with sample size $T=1000$ using MOESP and CCA on the original data (no pre.), on the preprocessed data (pre.) or including a constant term (incl.) in the calculations. Also the correlation (corr.) between the estimates (pre.) and (incl.) is given.

The input is Gaussian white noise with mean 1 and variance 1 . The noise $\varepsilon_{t}$ is chosen to be zero mean Gaussian white noise of variance 1 . The output $y_{t}$ is equal to

$$
y_{t}=\left(D+C(q-A)^{-1} B\right) u_{t}+\left(1+C(q-A)^{-1} K\right) \varepsilon_{t}+10
$$

Here $q$ denotes the forward shift operator. 1000 data sets of dimension $T=1000$ have been generated and for each data set the system is estimated

- using no preprocessing and no inclusion of a constant,

- mean corrected data and

- using the original data with the addition of a constant input variable.

$f=p=5$ has been chosen for the MOESP type of procedure and $f=5, p=15$ for the Larimore type of procedure, in all the trials. After the estimation the systems have been transformed to echelon canonical form. In both cases the CCA weightings have been used. The mean of the corresponding estimates for the MOESP type of procedures can be seen in Table 1.

It can be seen clearly, that the estimates without taking the nonzero constants into account is biased. It also can be seen, that the other two approaches give comparable means and are highly correlated, as is expected from the last section.

Comparing the mean of $y_{t}$ according to the estimated model to the actually estimated mean on the other hand shows a different picture: For the CCA procedure a correlation of 0.9989 is obtained, i.e. almost perfect agreement, while for the MOESP procedure the correlation is estimated as 0.0948 showing almost no linear dependence. This reaffirms the complications to be awaited for the derivation of the asymptotic distribution of the estimates of the components of $B$ and $D$ corresponding to $p_{t}$.

As a second example the same system is used, but the input is changed by adding a randomly weighted determinstic component and also the output is contaminated by a similar term:

$$
\begin{aligned}
& u_{t}=v_{t}+\beta x_{t} \\
& x_{t}=\left[\begin{array}{c}
t \\
\sin (0.5 \pi t) \\
\sin (0.22 \pi t) \\
1 \\
\sin (0.5 \pi(t-1)) \\
\sin (0.22 \pi(t-1))
\end{array}\right]
\end{aligned}
$$



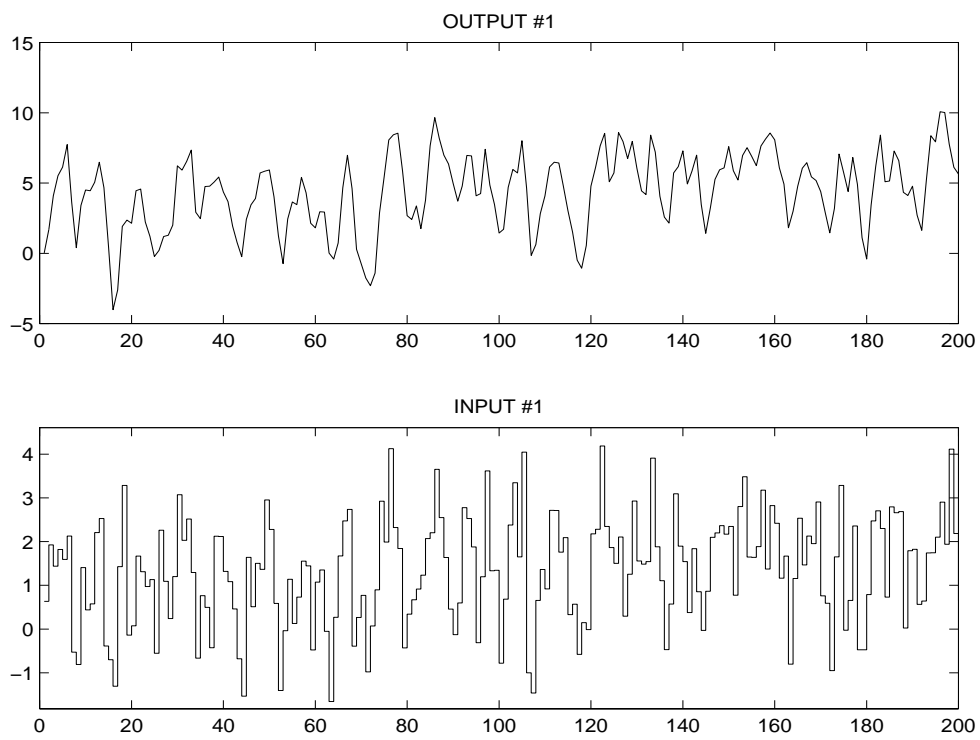

Figure 1: Observations 1 to 200 of a typical trajecory.

Here each component of $\beta$ is drawn from a $[0,1]$ uniform distribution. Also

$$
y_{t}=G(q) u_{t}+H(q) \varepsilon_{t}+\alpha x_{t}
$$

where

$$
\begin{aligned}
& G(q)=D+C(q I-A)^{-1} B \\
& H(q)=I+C(q I-A)^{-1} K
\end{aligned}
$$

The components of $\alpha$ are uniformely $[0,1]$ distributed. Finally $v_{t}$ and $\varepsilon_{t}$ are Gaussian zero mean and unit variance random processes. 1000 time series with these characteristics of sample size $T=1000$ are constructed. Parts of a typical input-output trajectory pair can be seen in Figure 1 . The estimation shows the same picture as the first example: Figure 2 shows the standard deviations of the estimates of the transfer function $D+C(q I-A)^{1} B$ at 50 points in the anguar frequency range $[\pi, \pi]$. Hereby $f=p=5$ was used and the MOESP type of procedures. The plot shows the standard deviation of the MOESP estimates using the preprocessed data and the standard deviation of the difference between the estimates obtained from the preprocessed data and the estimates obtained using the original data and including the additional variables, respectively. It can be seen clearly, that the difference in between the two approaches is of lower magnitude than the estimation errors themselves. For the CCA case the results are almost identical, therefore the presentation of the results is omitted.

\section{Conclusions}

In this paper we did derive the asymptotic properties of subspace procedures, when the data, which is used for the procedure, is preprocessed by removing trends and periodic components. It has been shown, that the preprocessing can alternatively be seen as the inclusion of additional input terms. This makes it possible to calculate the asymptotic variance of the estimates obtained with the procedure from the standard procedures for the subspace estimates. Also test procedures for more complicated hypotheses can be tested in the CCA framework. 


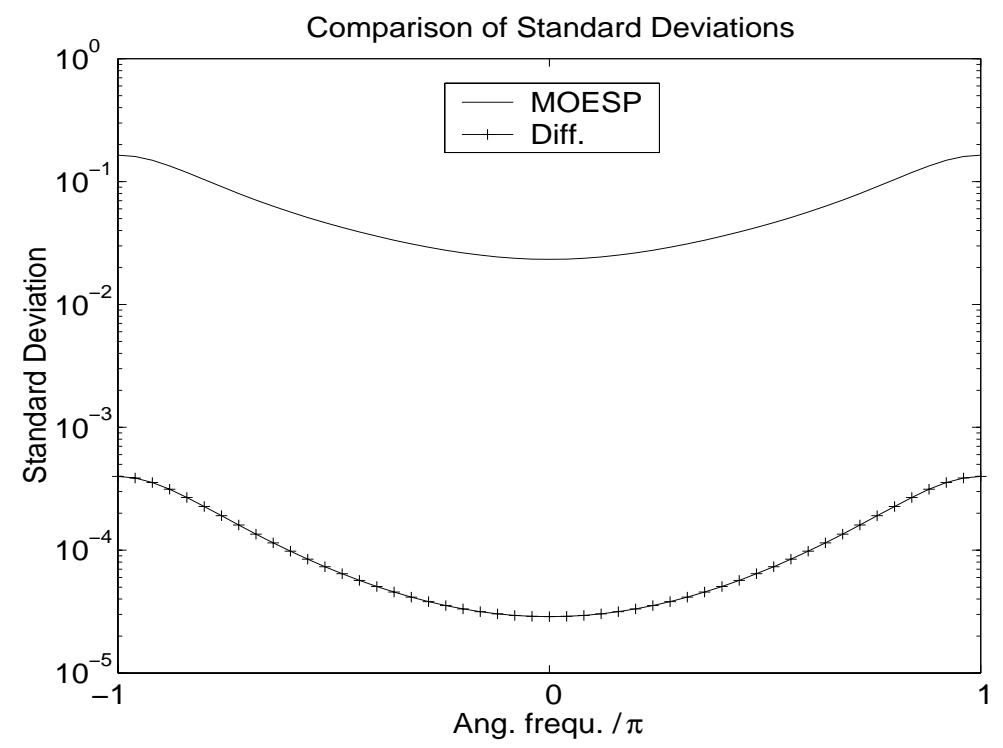

Figure 2: The plots show the standard deviation of the transfer function estimates obtained from the preprocessed data and $f=p=5(-)$ and the standard deviation of the difference of the two different procedures (-+-).

\section{References}

Bauer, D. (1998). Some Asymptotic Theory for the Estimation of Linear Systems Using Maximum Likelihood Methods or Subspace Algorithms. PhD thesis. TU Wien.

Bauer, D. and M. Jansson (2000). Analysis of the asymptotic properties of the MOESP type of subspace algorithms. Automatica 36(4), 497-509.

Bauer, D., M. Deistler and W. Scherrer (1999). Consistency and asymptotic normality of some subspace algorithms for systems without observed inputs. Automatica 35, 1243-1254.

Larimore, W. E. (1983). System identification, reduced order filters and modeling via canonical variate analysis. In: Proc. 1983 Amer. Control Conference 2. (H. S. Rao and P. Dorato, Eds.). Piscataway, NJ. pp. 445-451. IEEE Service Center.

Van Overschee, P. and B. DeMoor (1994). N4sid: Subspace algorithms for the identification of combined deterministic-stochastic systems. Automatica 30, 75-93.

Verhaegen, M. (1994). Identification of the deterministic part of mimo state space models given in innovations form from input-output data. Automatica 30(1), 61-74. 